\title{
PNEUMATOSIS INTESTINALIS - A RARE CLINICAL FINDING REQUIRING MULTIMODALITY TREATMENT
}

\author{
Namra Urooj ${ }^{1}$, Mahin Janjua ${ }^{2}$, Awais A. Malik ${ }^{3}$, Shahid Khattak ${ }^{1}$, Aamir A. Syed $^{1}$ \\ ${ }^{1}$ Department of Surgical Oncology, Shaukat Khanum Memorial Cancer Hospital and Research Centre, Lahore, \\ Pakistan, ${ }^{2}$ Aga Khan University, Karachi, Pakistan, ${ }^{3}$ Department of Surgery, Lahore General Hospital, Lahore, \\ Pakistan
}

Received: 11 August 2018 / Accepted: 26 September 2018

\begin{abstract}
This is a case report of pneumatosis intestinalis (PI), which has traditionally been associated with immediate operative intervention and a high mortality rate. We present a case of ulcerative colitis (UC) that developed PI and were managed at our hospital. A 29-year-old male with a known history of UC presented with an acute exacerbation. A week into his treatment, PI developed. Emergency total colectomy with end ileostomy was performed. Postoperatively, he remained critically ill and expired during the same hospitalization after 45 days.
\end{abstract}

Key words: Adalimumab, pneumatosis intestinalis, ulcerative colitis

\section{Introduction}

Pneumatosis intestinalis (PI), defined as gas within the bowel wall, is an uncommon radiographic sign which represents a wide spectrum of diseases and a variety of underlying diagnoses. ${ }^{[1]}$ Because its aetiology can vary greatly, management of PI ranges from surgical intervention to outpatient observation. ${ }^{[2-4]}$ Since PI is infrequently encountered, clinicians may be unfamiliar with its diagnosis and management. We present a case of ulcerative colitis (UC) that developed PI and were managed at our hospital.

\section{Case Report}

A 29-year-old male presented to the gastrointestinal (GIT) clinic with 10 weeks history of diarrhoea, weight loss and fever. During his workup to establish diagnosis, endoscopic biopsies were taken from all parts of colon which revealed histopathological diagnosis of inflammatory bowel disease. He was started on prednisolone $45 \mathrm{mg}$ and mesalazine $1600 \mathrm{mg}$ BD. 3 months later, he presented with acute exacerbation of UC with a history of 20-22 episodes of watery stools per daily intermittent bloody diarrhoea

Correspondence: Dr. Namra Urooj, Department of Surgical

Oncology, Shaukat Khanum Memorial Cancer Hospital and Research

Centre, Lahore, Pakistan.

Email: namra_urooj@yahoo.com and significant weight loss. He was started on medical management including intravenous fluids, antibiotics and total parenteral nutrition. His medications were switched to sulphasalazine, azathioprine and adalimumab.

After 1 week of conservative treatment, his condition started deteriorating and he developed hypotension, tachycardia and major electrolyte abnormalities along with altered state of consciousness. He also developed extrapyramidal involuntary movements and his Glasgow Coma Scale (GCS) score declined to 8/15. The patient was transferred to the intensive care unit (ICU) and required endotracheal intubation for his deteriorating condition.

A magnetic resonance imaging scan of the brain reported non-communicating moderate hydrocephalus with possible obstruction at the level of aqueduct. This was managed by urgent placement of an external ventricular drain.

The patient remained critically ill requiring ventilatory and vasopressor support. 4 days later, he was noted to have developed subcutaneous emphysema along the chest and anterior abdominal wall. A contrast-enhanced computed tomography (CT) scan of the abdomen was performed that showed extensive subcutaneous surgical emphysema along the anterior and posterior chest 
walls extending up to neck and bilateral upper limbs superiorly and lateral abdominal walls inferiorly. There was gross pneumomediastinum with air extending down to retroperitoneum with free air in retropancreatic area. In addition, small bilateral pneumothoraces were noted along with bilateral cavitary lesions with internal mural nodule concerning for fungal infection. This was managed with bilateral chest drain insertion and empiric antifungal therapy with voriconazole.

He showed some clinical improvement but could not be weaned off the ventilatory support due to persistent respiratory distress.

A ventriculoperitoneal shunt was performed for his persistent hydrocephalus. His blood cultures grew Gramnegative bacteria.

A repeat contrast-enhanced CT scan of the abdomen demonstrated new development of PI involving the ascending colon, extending to the hepatic flexure [Figure 1]. Immediate emergency total colectomy with end ileostomy was performed. He remained on ventilator support postoperatively. Histopathology report of the specimen was consistent with ulcerative colitis.

The patient stayed in ICU for 45 days postoperatively. He was started on enteral feed initially but had to be stopped due to minimal stoma output and high nasogastric output. His GCS dropped to 2/10(T). There was no radiological evidence of definite mechanical obstruction. The patient remained on ventilator for 77 days and expired on the $100^{\text {th }}$ day of admission due to a cardiac arrest. Autopsy was refused by the family and was not performed.

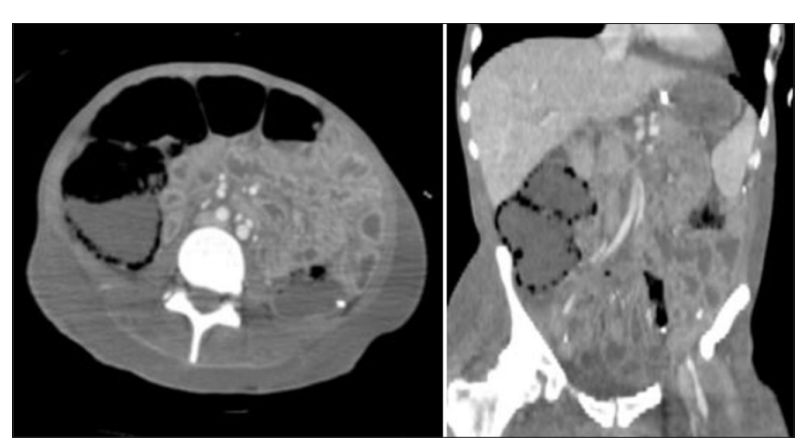

Figure 1: Computed tomography showing air in the wall of the ascending colon (axial and coronal view)

\section{Discussion}

Pneumatosis refers to the presence of gas or air at an abnormal location in the body. ${ }^{[1]} \mathrm{PI}$ also refers to the presence of air or gas in the wall of the small or large intestine ${ }^{[1-5]}$ It is also known as intramural gas, pneumatosis cystoides intestinalis, etc. PI is a clinical sign and not a disease, usually first diagnosed on radiographic study. Interpretation of this sign must be made relative to the clinical condition of the patient and other associated signs and symptoms as well as laboratory findings ${ }^{[6]}$ to decipher a benign disease from a life-threatening one.

PI is a relatively rare finding having an overall incidence of $0.03 \%$ among the general population based on autopsy findings. ${ }^{[3]}$ However, recently, the overall incidence has increased to $0.3 \%$ due to extensive use of CT scan in recent years. In the past literature, PI has been described in association with a number of GIT disorders, including infection, obstruction or ischemia. Other than GITrelated issues, it has also been reported with chronic obstructive pulmonary disease, connective tissue disorders, organ transplantation, leukaemia and various states of immunodeficiency. ${ }^{[5-7]}$

PI has been classified into primary and secondary types. Primary PI is when the cause of PI is not known i.e., idiopathic, whereas, secondary PI is termed when there is an underlying disease that may have led to this condition. Primary PI accounts for only $15 \%$ of the cases, whereas secondary PI, being more common, accounts for $85 \%$ of cases. ${ }^{[6]}$ Secondary PI can be associated with a number of diseases, including inflammatory bowel disease; however, the incidence is low.

In our case, the cause of PI could be multifactorial. PI was preceded by an episode of bilateral pneumothorax and subcutaneous emphysema which could make it a likely cause. Fungal infection is one possibility... also the baseline pathology was UC which could still be the primary cause. Furthermore, a few cases have been reported in literature associated with UC. ${ }^{[9]}$ But to say with certainty what caused the PI in this patient is not an easy task.

Our patient presented with 10 weeks history of diarrhoea, weight loss and fever. If literature review 
is taken, patients with PI mostly present with a history of diarrhoea, abdominal pain and discomfort, abdominal distension, bloody stools, constipation, loss of weight and tenesmus. In some cases, PI may be an incidental finding as is the case in benign causes of PI like PI associated with pulmonary disease where no particular treatment is needed. ${ }^{[7]}$ However, some patients may present in a critical condition and these patients are mostly among those who have PI due to a life-threatening underlying disease, such as mesenteric ischemia.

On CT scan, our patient was diagnosed as having PI. Among the cases reported in literature, CT scan has remained a sensitive modality in diagnosing the PI. ${ }^{[6,9]}$

Another possible cause of PI could be adalimumab. The patient was on this drug for a week when he developed a hydrocephalus followed by PI. Although not reported with adalimumab, other molecular targeted therapies have been shown to be associated with PI. ${ }^{[9]}$ We cannot for sure establish a link between the two, but its role cannot be ruled out.

\section{Conclusion}

PI is a rare entity. It has not been labelled as a disease but a sign. Careful observation followed by aggressive intervention for a patient who deteriorates is the main management strategy. A multidisciplinary approach with careful patient monitoring and a low threshold for surgery can help reduce mortality from this complication.

\section{Conflict of Interest}

The authors declare that they have no conflict of interest.

\section{References}

1. Pneumatosis. The Free Dictionary.com. 2017. Available from: http://www.medical-dictionary.thefreedictionary.com/ pneu matosis. [Last cited 2017 Mar 2017].

2. Knechtle SJ, Davidoff AM, Rice RP. Pneumatosis intestinalis. Surgical management and clinical outcome. Ann Surg 1990;212:160-5.

3. Heng Y, Schuffler MD, Haggitt RC, et al. Pneumatosis intestinalis: A review. Am J Gastroenterol 1995;90:1747-58.

4. Morris MS, Gee AC, Cho SD, et al. Management and outcome of pneumatosis intestinalis. Am J Surg 2008;195:679-82.

5. Hanna P, Kassir R, Tarek D, et al. Pneumatosis cystoidis intestinalis presenting as bowel perforation: A rare entity. Int J Surg Case Rep 2016;20:7-9.

6. St Peter SD, Abbas MA, Kelly KA. The spectrum of pneumatosis intestinalis. Arch Surg 2003;138:68-75.

7. Doumit M, Saloojee N, Seppala R. Pneumatosis intestinalis in a patient with chronic bronchiectasis. Can J Gastroenterol 2008;22:847-50.

8. Schneider JA, Adler DG. Pneumatosis coli in the setting of severe ulcerative colitis: A case report. Dig Dis Sci 2006;51:185-91.

9. Shinagare AB, Howard SA, Krajewski K M, et al. Pneumatosis intestinalis and bowel perforation associated with molecular targeted therapy: An emerging problem and the role of radiologists in its management. Am J Roentgenol 2012;199:1259-65. 